\title{
O N IMPROVING THE FAULT DETECTION AND RELIABILITY IN FDDI NETWORKS
}

\author{
R. Radhakrishna Pillai, Anand Hardi and A. Selvarajan \\ Department of Electrical Communication Engineering \\ Indian Institute of Science \\ BANGALORE - 560 012, India.
}

\begin{abstract}
FDDI (Fiber Distributed Data Interface) is a $100 \mathrm{Mbps}$ token ring network with two counter rotating optical rings. Various possible faults (like lost token, link failures etc.) are considered and fault detection and the ring recovery process in case of a failure and the reliability mechanisms provided are studied. We suggest a new method to improve the fault detection and ring recovery process. The performance improvement in terms of station queve length and the average delay is compared with the performance of the existing fault detection and ring recovery process through simulation. We also suggest a modification for the physical configuration of the FDDI networks within the guidelines set by the standard to make the network more reliable. It is shown that, unlike the existing FDDI network, full connectivity is maintained among the stations even when multiple single link failures occur. A distributed algorithm is proposed for link reconfiguration of the modified FDDI network when many successive as well as simultaneous link failures occur. The performance of the modified FDDI network under link failures is studied through simulation and compared with that of the existing FDDI network.
\end{abstract}

\section{Introduction}

FDDI is an ANSI (American National Standards Institute) standard for 100 Mbps data communication using fiber optic medium in a ring configuration. Its operation is based on a timed-token protocol which faciliuctes a flexible priority scheme for transmission of synchronous and asynchronous packets

[4]. The basic ideas of the timed-token protocol were first presented by Grow[6]. The nodes on the network choose, in a distributed fashion, a target token rotation time(TTRT). A station can use the token to transmit asynchronous traffic only if token rotation time(TRT) (TRT is the time elapsed when the token arrives the station since the token is last seen by the station) is less than TTRT. Ulm[11] investigated the protocol described by Grow and determined its sensitivity to various parameters. Sevick and Johnson[3] have analytically proved the upper bounds on the average TRT and maximum TRT. Dykeman and Bux[1] studied the relationship between the FDDI MAC: parameter settings, the ring configuration and the performance of the asynchronous priority levels. Johnson[12] investigated the reliability mechanisms provided in the FDDI protocol.

This paper focuses on some issues on fault detection and reliability of FDDI networks. Modifications have been proposed for fault detection and physical reconfiguration of FDDI networks and the performance characteristics are analyzed based on simulation of the network operation. This paper is organized as follows.

Section 2 deals with the existing reliability mechanisms in FDDI. Section 3 discusses the proposed fault detection method and section 4 explains the modified physical configuration of FDDI network with a distributed algorithm for ring reconfiguration. Section 5 presents the performance of the modified FDDI network and the conclusions drawn are presented in section 6 .

\section{Existing Reliability Mechanisms in FDDI}

The common errors occurring in local area networks (LAN) can be classified into two category; protocol related errors, and errors due to the failure of a physical resource. The former one includes lost token, frames circulating indefinitely in the ring, fault with late-ct, dual or multiple tokens, dual address problem etc. and the latter one consists of break in the ring, station failure, fault with various timers etc.

The FDDI protocol features several mechanisms which automatically detect and initiate ring recovery. If the error is protocol related then the ring recovery process will correct it and if it is due to a physical failure then the ring automatically reconfigures so as to bypass the failed resources. Physical reliability provisions employed in FDDI are: (i) Station bypass switch (to bypass any station from the ring, if required), (ii) Counter rotating rings (a second ring, a standby for the primary ring, can be used to maintain full connectivity and concurrent transmission), and (iii) Concentrators (this is used to attach class B stations to the ring and allows any combinations of stations to be switched out of the ring).

Automatic fault detection, isolation and correction are accomplished in a manner transparent to the user[12]. Frame stripping is done by the source station by recognizing the source address one cycle after the transmission. The maximum token rotation time is 2TTRT2]. The number of expirations of the TRT is indicated by the counter, late-ct. The expiration of TRT when late $-c t<>0$ initiates the ring recovery process.

Physical reconfiguration in case of a station failure is achieved by bypassing a failed class A statioii through the bypass switch and a failed class B station through the concentrator. In case of a link failure, the ring is reconfigured using operational links of both the rings. The two rings are folded into a one ring, which is approximately twice as long, maintaining full connectivity. Multiple link failures divide the original link into two or smaller rings, if the failed link belongs to a class A station.

\section{Fault Detection with Valid Transmission Timer}

The basic concept behind this idea has been suggested in [12], but its application has not been explored true to its worth. The valid transmission timer (TVX) maintains proper operation of the ring by ensuring that the transmission on the channel is meaningful. The TVX is reset each time a "non-restricted" token is received or a valid frame is received (Tokens for the general operation of the ring are non-restricted; two stations may hog the channel for asynchronous communication on issuing a "restricted" token). TVX is reset to the maximum value $t v$ it takes for a valid frame or a non-restricted token to return to a station. Maximum delay occurs when the stations repeat the token (i.e., when it does not transmit) and subsequently that token is captured by some other station to transmit a frame. Hence, $\mathrm{tv}=D+c+a$ where

D - ring latency (including the station delays), c - token capture delay, and a - a frame transmission time. 
When a station has finished using a restricted token, the last one is supposed to issue a non-restricted token and the normal operation of the ling is restored. It is mentioned by Johnson [12] that if a station erroneously issues a restricted token at the end of network hogging, TVX is not reset and the expiration of TVX initiates ring recovery. This prevents restricted token from circulating forever and thus blocking access to the ring. But this overlooks the fact that synchronous stations are transmitting valid frames even during restricted mode and hence TVX may not expire at all. Thus the fault may go undetected.

To circumvent this problem, we suggest that during the restricted token operation, TVX he reset to a value $T T R T+a$ and it is reset only when a valid asynchronous frame is received. Where $\mathrm{a}=$ an asynchronous frame duration. TTRT $+a$ is the maximum time it takes a station to receive an asynchronous frame. During the restricted mode of operation, synchronous stations transmit for a maximum duration of TTRT and the asynchronous stations involved in network hogging has a frame to transmit in every token cycle, After the completion of the restricted token mode, if the station erroneously issues a restricted token again, the TVX will expire as no asynchronous frames exist. This initiates the ring recovery.

During normal non-restricted mode of operation, a station need not wait for a time $2 T T R T$ to expire (when token is lost or a link fails) before initiating ring recovery. Truncated frames will not reset TVX. Since $t v$ is far less than $T T R T$, fault detection and hence the overall recovery process can be made more efficient. Simulation results for fault detection with TVX is discussed in section 5 along with performance characteristics of a modified FDDI network.

\section{A Modified Physical Configuration for FDDI Network}

A link failure which initiates a physical reconfiguration implies a fault in the transmitter of a station, receiver of the downstream station or in the link in between the stations, In case of a link failure, the standby ring or a single ring formed by folding the two rings is used to maintain full connectivity. From reliability point of view one would prefer to lay the two rings separately and the two PHY entities he maintained separately. in such a situation, it is unlikely that both PHY entities of a station fail simultaneously or both the links in the segment between two stations fail simultaneously. Hence we can define two kinds of link failures viz.

a) Single fault : When a link between two stations fail either in the outer ring or in the inner ring, but not in both.

b) Double fault : When both the inner and outer links in the ring segment between any two adjacent stations fail simultaneously or successively.

It will be interesting to examine the fault tolerance of an FDDI network with both rings in the same direction. When single faults occur successively or simultaneously the operational link (inner or outer) can still be used to maintain full connectivity among the FDDI stations. But in the former case of two counter rotating rings two single fault would have resulted in two rings folding into a single ring and subsequent single fauits result in two or more disjoint rings, each of them providing only partial connectivity. However, when a double fault occurs, inevitably the two ring should be folded into one ring to retain full connectivity which necessitates two counter rotating rings. These two requirements can be satisfied by using an optical switch or coupler in the inner ring capable of interchanging the connections to receiver and transmitter at each station. This switch can be provided at PMD of secondary ring as shown in Fig. 1.

Initially the two rings are in opposite direction and when faults occur, the link directions are reversed if necessary to maintain maximum connectivity.

\subsection{Reconfiguration Algorithm}

Here we present a distributed algorithm which carries out the reconfiguration of the network effectively as long as one link in each of the ring segment is operational. it is assumed that the outer ring is the primary ring and all the modifications suggested earlier in this section do exist in the ring. The algorithm is described below. a) Stations which do not recelve token or valid frames within expected time initiate ring recovery. Call these stations as RBCOVERY stations. However, if ring recovery fails, they tme-out and send SUPERVISORY frames to their downstream stations.

b) Stations acknowledge the receipt of SUPERVISORY frames to their upstream stations on the :secondarylink after activating the secondary link transceiver. They use $A C K$ NOWLEDGMEN'I frames for this purpose. Also they repeat. the SUPERVISORY frames to their downstream stations.

If a station is already using the secondary link for its communcation with the downstream station (i.e. if the dowustream segment has already been reconfigured in some previous process), it sends the acknowledgment to the upstream station by reversing its transceiver connections using the optical switch, then switches back to send the SUPERVISORY frame to the downstream station and then again reverses its connections. The downstream station also switches its connection and sends the ACKNOWLEDGMENT frame.

c) Stations which do not receive any acknowledgment, from the downstream stations (called INSPECTOR stations) time-out and initiate INSPECTION frames on the secondary link.

d) All stations which receive the INSPECTION frames repeat, it on the secondary link. Also RECOVERY stations, which initiated the reconfiguration in the present case or in some previous reconfiguration process, on receiving the INSPECTION frame interchange the connections to their transmitter and receiver on the secondary link using the optical switch.

e) If the INSPECTOR station (which has issued the INSPECTION frame) receives INSPECTION frame sent by any station (including the one sent by itself), they remove the frame from the ring and then interchange the connections to their transmitter and receiver on the secondary ring using the optical switch. Stations which were INSPECTOR stations in some previous process and now using only the inner link for the downstream communication also switch their connections. INSPECTOR stations which do not receive any INSPECTION frame do not reconfigure.

f) All INSPECTOR stations time-out after a cycle time and transmit, CHECK frames. INSPECTOR stations which have reconfigured as explained in step(e) send the frames on their secondary link but all other INSPECTOR stations (which have not reconfigured in step (e)) transmut on their primary link. Stations other than the INSPECTOR stations repeat the check frames on their primary link

g) If INSPECTOR stations receive back frames after a cycle, ring recovery is initialised by these stations. Otherwise steps (h) and (i) are carried out.

h) INSPECTOR stations loop back (i.e., primary and secondary links at theiir nodes) in the upstream direction. Before doing so they send frames to their downstream stations asking them to loop back.

i) RECOVERY stations which do not receive any check frame within a cycle time since it last received the INSPECTION frame, reconfigure and loop $\Omega$ back in the downstream direction. Also the stations (including RECOVERY stations) which receive frames from their upstream stations in step (h) reconfigure and loop back in the same way. Then INSPECTOR stations initiate rin recov ry. One or more smaller rings are formed. RECOVER and INSPECTOR stations of same previous reconfiguration, if any, also loop back in the same way as given in steps $(\mathrm{h})$ arid (i).

Each of the SUPERVISORY frame, ACKNOWLEDGMENT frame, INSPECTION frame and CHECK frame has unique frame control(FC) field. SUPERVISORY and CHECK frames arc transmitted on the primary ring while ACKNOWLEDGMENT frame and INSPECTION frames arc transmitted on the inner ring. The state diagram corresponding to the ring reconfiguration algorithm discussed above is shown in Fig. 2. Eventhough the addition of an active component, the optical switch, can be viewed as a cause for degradation in reliability of the network, the overall reliability cannot be worse than the reliability of the normal FDDI network without the switch. 


\section{Simulation Model and Results}

The effects of various faults and the corresponding recovery pro cess/reconfiguration on the overall network performance is studied through simulation. The faults which significantly affect the network operations have been considered here to justify the improvements suggested in section 3 and 4 are: (i) token loss, (ii) single link failure, and (iii) double link failure

The simulation program was written in SIMULA and executed on DEC 1090 computer. Only one fault is assumed to occur in each simulation study and is made to happen in the middle of the simulation period. A simulation model-with the following assumptions is used.

All stations are equally spaced, transmission is error free, in each station the buffer size is sufficiently large, data stations have exponentially distributed packet inter-arrival time with equally sized packets, a station can transmit maximum of only one packet each time it has access to the network, voice and data stations are separate, and PCM coding for voice.

\subsection{Simulation Parameters}

\subsubsection{Network Parameters}

Network capacity is $100 \mathrm{Mbps}$, ring length is $10 \mathrm{~km}$ with propagation delay of the medium as $5 \mathbf{s} / \mathbf{k m}$, voice or data packet length is 400 bytes, and TTRT is $25 \mathrm{~ms}$ (decided by the voice packet length).

\subsubsection{Station Parameters}

$\therefore$ Latency of a physical connection is $6 \mathrm{C}$ bits, transmitter idle time after token capture is $0.0035 \mathrm{~ms}$, mean inter-arrival time of packets to a station is $1.0 \mathrm{~ms}$ (Corresponds to a data load per station of $3.2 \mathrm{Mbps}$ ) and the number of active stations is varied to get different load conditions, interarrival time of voice packets at a station is $50 \mathrm{~ms}$, and the number of voice stations is fixed as 50 .

\subsection{Performance Characteristics and Discussions}

\subsubsection{Fault Detection and Recovery}

As explained in section 3, the overall efficiency of fault detection and recovery can be improved by using valid transmission timer(TVX) to detect the errors. The TVX is reset each time a valid frame or a non-restricted token is received. Hence TVX is loaded with a much smaller value than token rotation time(TRT). Time-out of TVX initiates ring recovery. Once the fault is detected, the effect of recovery process on the average packet delay is negligible as shown in Fig. 3. This is expected as the recovery process involves at most two cycles to decide the highest priority station and two more cycles for initialization before normal operations are restored.

It is the fault detection which requires more time and affects the efficiency of the overall recovery process. Fig. 4 and 5 show that the average packet delay is considerably longer for detection with TRT. Fig. 5 also shows that the average packet delay increases with increasing value of TTRT for detection with TRT whereas it is independent of TTRT for detection with TVX. This is because a station waits for TRT to expire twice before initiating recovery even when the token is returning to a station in time much less TTRT. However, TVX always looks for a valid frame or a non-restricted token.

For a given arrival rate, the increase in average packet delay is because of the increased waiting time in the queue. The maximum queue length in each station increases with increasing detection period. Fig. 6 and 7 show that the average maximum queue length over all the stations (when fault occurs) increases with increasing value of TTRT for detection with TRT, whereas the average maximum queue length for detection with TVX is almost constant and much smaller than that in the case of TRT. This implies smaller fuffer requirement at a station when the offered load is up to about $70 \%$ Also for a given buffer size limit, with TVX fault detection, the station can afford a higher packet arrival rate than in the case of fault detection with TRT. Maximum voice and data packet delay is also considerably less for detection with TVX as shown in Fig. 8.

The use of TVX is really advantageous when the offered load is not very high and so when the mean inter packet arrival time is greater, than the mean token time. As shown in Pig. 10, when the packet arrival rate is very high the network performance on fault detection with TVX is close to that on fault detection with TRT, both resulting in large waiting time.

\subsubsection{Link Failures}

The effect of the modified ring configuration on the network performance is discussed here. Improved performance is observed when TVX is used for fault detection. Fig. 9 shows that when a single fault occurs in both the rings, the average end-to-end delay is considerably larger for the normal FDDI network compared with the modified FDDI network making use of the inner link and detecting faults with TVX. The latter also shows a constant delay upto $60 \%$ load. The increased delay in normal FDDI network is because that the two rings loop back and a single ring approximately twice the length of the original ring is formed. This increases the propagation delay and hence the waiting time. Fig, 11 shows that maximum delay for voice and data packets are also considerably less for modified FDDI network when a single fault occurs

The modifications suggested €or FDDI network is more effective (in addition to maintaining full connectivity when single fault occurs) when the ring length is made larger. As Fig. 12 shows, the performance of normal FDDI network degrades considerably as the ring length increases. This is because the token rotation time for the same offered load has increased and hence larger waiting rime for the packets. The performance worsens when mean token rotation time on folding the rings exceeds the packet arrival time a.t a station. Fig. 13 shows the performance characteristics when a doublc fault occurs in which case both the normal and the modified FDDI networks loop back. However, the one employing fault detection with TVX gives improved performance at low/medium loads (upto 70\%). But, as Fig. 14 shows, the performance characteristics of FDDI network with double fault, detecting the fault with TVX does not yield considerable improvement over the one detecting the faults with TRT when the ring. length is made much larger $(60 \mathrm{~km})$ such that the mean packet arrival time at data station becomes les than the mean token rotation time. Waiting time increases for the packets and the delay increases considerably.

\section{Conclusion}

A novel method to improve the performance of the FDDI network during fault detection process, employing the valid transmission timer is suggested in this paper. A new reconfiguration method employing an optical switch in the secondary ring to improve the fault tolerance of FDDI networks under the occurrence of multiple fault is also suggested. The performance of the network with these modifications is studied and found to be superior to the normal FDDI network performance. The cost/performance comparison of these two models have to be carried out. Also the mathematical analysis of the reliability of these FDDI network models in case of various link failures give scope for future work

\section{References}

[1] D. Dykeman and W. Bux, "Analysis and Tuning of the FDDI Media Access Control Protocol," IEEE JI. on Selected Areas in Commn. Vol.6, No.6, ,pp. 997-1010, July 1968.

[2] M. J. Johnson, "Proof that Timing Requirements of the FDDI Token Ring Protocol are Satisfied," IEEE Trans. Commn., Vol. COM-35, No.6, pp. 620-625, June 1967.

[3] K. C. Sevick and M. J. Johnson, "Cycle Time Properties of the FDDI Token Ring Protocol," IEEE Trans. Soft. Eng., Vol. SE-13, No. 3, pp. 376-385, March 1987

[4] F. E. Ross, "An Overview of FDDI: The Fiber Distributed Data Interface," IEEE Jl. on Selected Areas in Commns., Vol. 7, No.7, pp. 1043-1051, Sept. 1989. 
[5] W. Bux, "Token-Ring Local-Area Networks and Their Performance,!' Proc. IEEE, Vol. 77, No.7, pp. 238-256, Feh 1983

[6] R. M. Grow, "A Timed Token Protocol for Local Area Networks," Presented at Electro '82, Token Access Protocols, Paper 17/3, May 1982.

[7] R. Jain, "Performance Analysis of FDDI Token Ring Networks: Effects of Parameters and Guidelines for Setting TTRT." Dig. Eqpt. Corpn. Report DEC-TR-655, Sept. 1989

[8] Fiber-Distributed Data Interface (FDDI) Token Ring Media Access Control (MAC), American National Standard, ANSI X3.139-1987, Nov. 5,1986

[9] Fiber-Distributed Data Interface (FDDI) Token Ring Physical Layer Protocol Control (PHY), American National Standard, ANSI X3.148. 1988, Jun. 30, 1988

[10] Fiber-Distributed Data Interface (FDDI) Token Ring Physical Medium Dependent (PMD), American National Standard, ANSI X3T9/85X3T9.5/84-48, Jun. 10.1986

[11] J. M. Ulm," A Timed Token Ring Local Area Network and its performance Characteristics," Proc. 7th IEEE Conf. Local Computer Networks, pp. 50-56, Feb. 1982.

[12] M. J. Johnson, "Reliability Mechanisms in the FDDI high bandwidth protocol," Computer Networks and ISDN Systems, Vol. 11, No. 2, pp. 121-131, Feb. 1986.

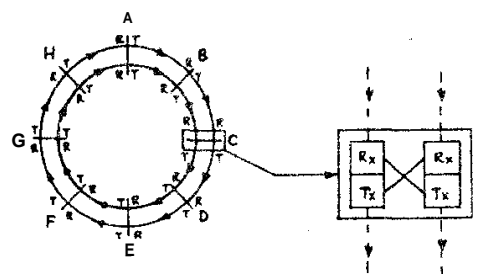

FDDI RING WITH INNER RING DIRECTION EEVERSED
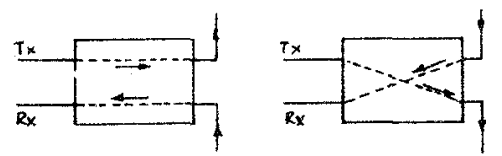

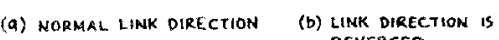

OPTICAL SW:TCH

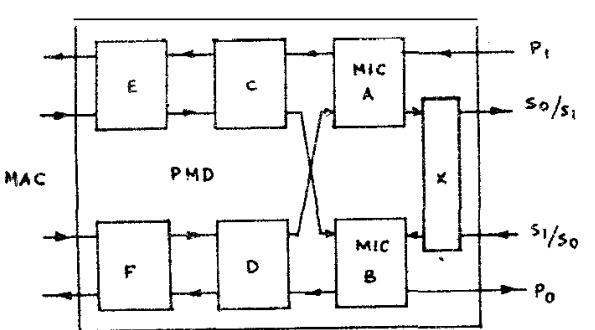

Fig. 1: PMD WITH AN OPTICAL SWITCH $(x)$

Mic - MEDIA INTERFACE CGMNECTOR $X$ - OPTICAL SWITCh

C - PRIMARY OPTICAL SWITCH PI-PRIMARY IN

D - SECONDARY OPTICAL SWITCH ST-FECONDARY IN

E, F - TRAMSMITTER AND RECEIVER Po-PRIMARY OUY

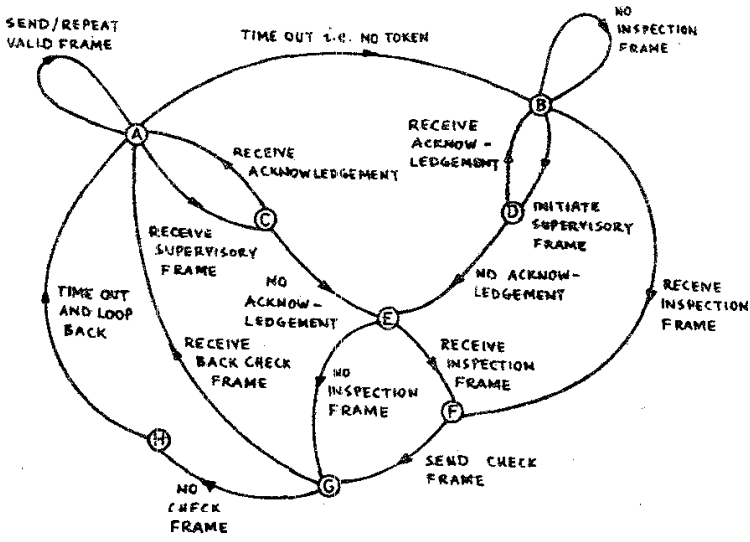

Fig. 2. State Diagram Corresponding to thr King Reconfiguration Algoritbro

A - Normal operating state, $\mathrm{B}$ - Recovery stabion. C and D Loukisg for an ACKNOWLEDGEMENT from the fallowing station, E- INSPECTOR statton, F - Changroves to secondary link for commuaication, $G$ - INSPECTOR station sending CHECK frame. H - Forming two or more snialler rings

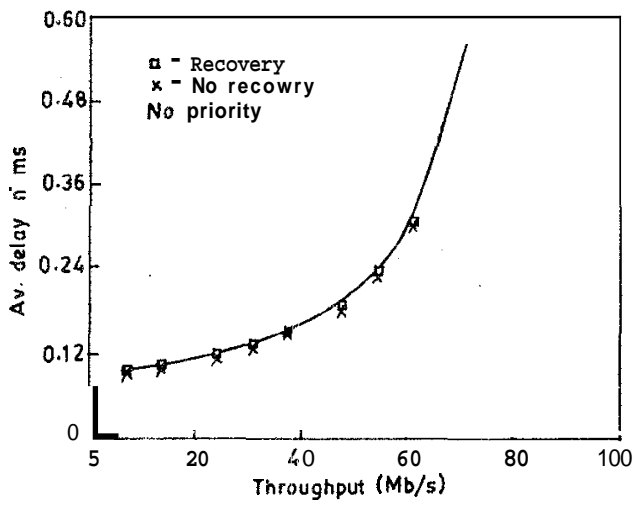

Fig.3: Effect of Ring Recovery Process on the Delay vs. Throghput Characteristics (Lust token)

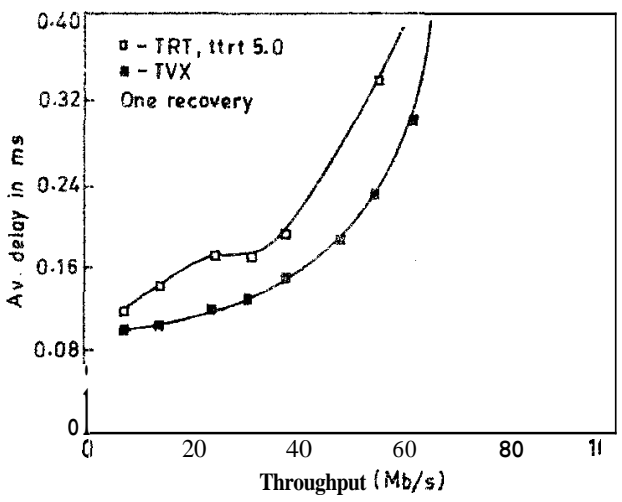

Fig. 4: Delay vs. Throghput Chatacteristics under Fulit De. tection (Lost token) 


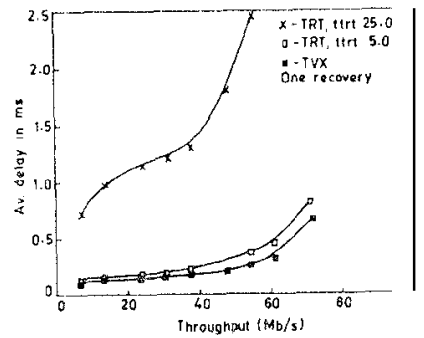

Fig.5: Effect of 'TTRT on Delay vs. Throghput Characteris tics under Fault Detection (Lost token)

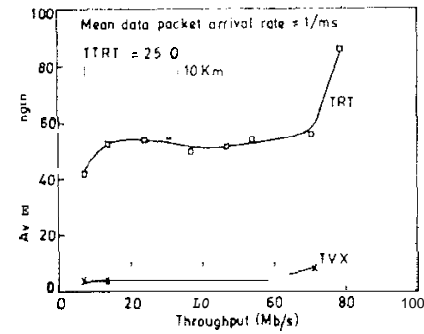

Fig.6: Average max queue length under Fault Detection (Lost token)

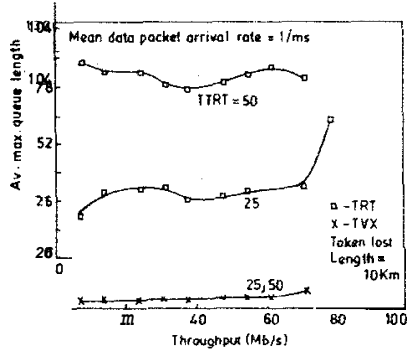

Fig.7: Effect of TTRT on Average max. queue length with Fault Detection (Lost token)

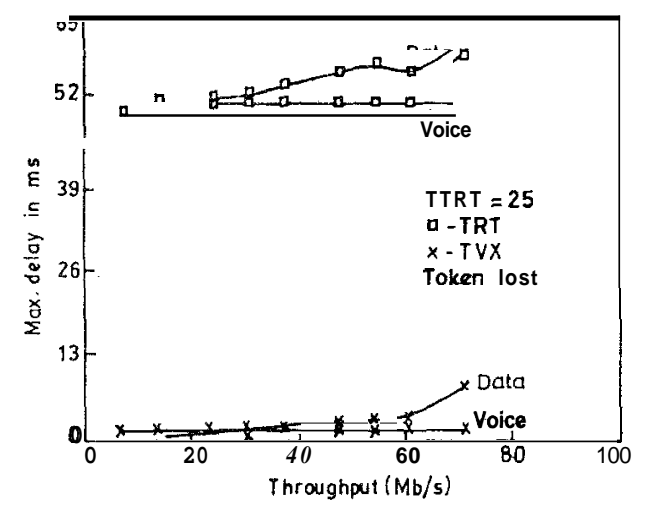

Fig.8: Max. Voice and Data Packet Delay vs Throghput Characteristics with Fault Detection (Lost token)

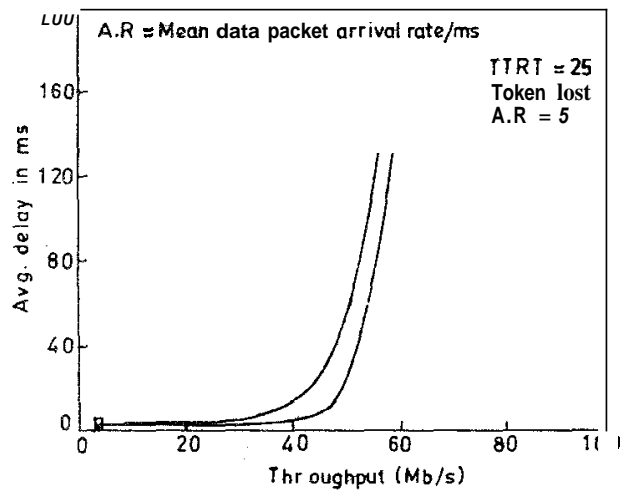

Fig 10: Delay vs Throghput Characteristics at high mean Data Packet arrival rate'(Single Fault)

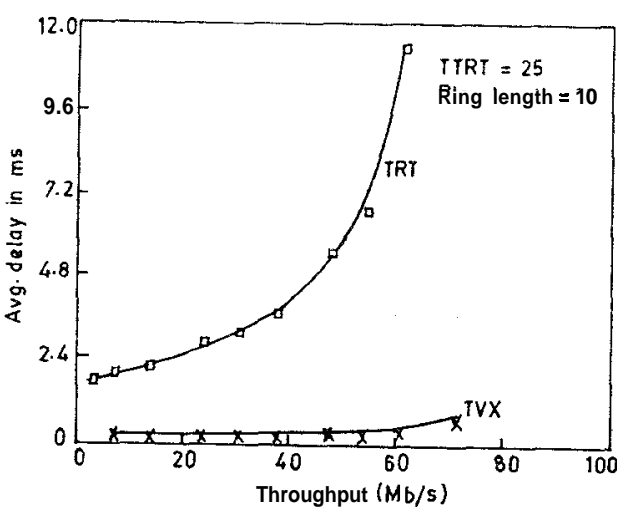

Fig.9: End-to-end Delay vs. Throghput Characteristics with Fauit Detection (Single Fault)

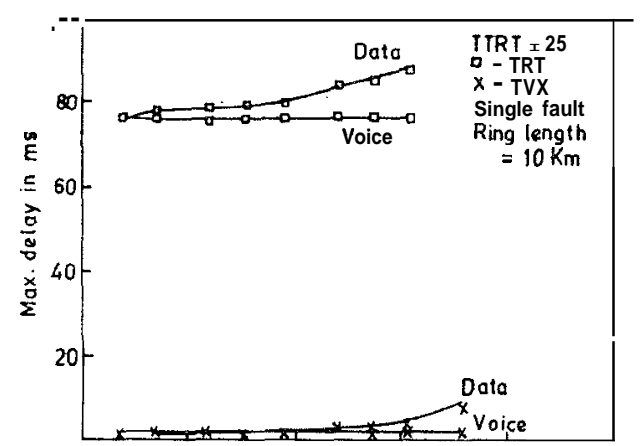

Fig.11: Max. Voice and Data Packet Delay vs. Throghput Characteristics with fautt detection (Single Fault)

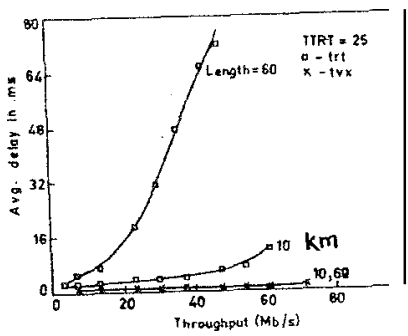

Fig. 12: Effect of Ring length on Delay vs Throughput Characteristics with fault detection (Single Fault)
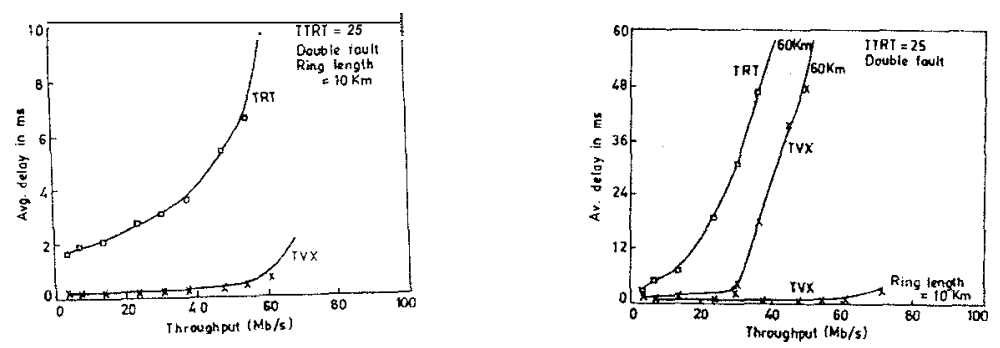

Fig. 13: Delay vs. Througliput Characteristics (Double Faniti
Fig 14: Effect of Ring length on Delay is. Througliput 